\title{
BMJ Open Risk scores identifying elderly inpatients at risk of 30-day unplanned readmission and accident and emergency department visit: a systematic review
}

Camille Schwab, ${ }^{1,2}$ Patrick Hindlet, ${ }^{1,3,4}$ Brigitte Sabatier, ${ }^{2,5}$ Christine Fernandez, ${ }^{1,3,4}$ Virginie Korb-Savoldelli ${ }^{2,4}$

To cite: Schwab C, Hindlet P, Sabatier B, et al. Risk scores identifying elderly inpatients at risk of 30-day unplanned readmission and accident and emergency department visit: a systematic review. BMJ Open 2019;9:e028302. doi:10.1136/ bmjopen-2018-028302

- Prepublication history and additional material for this paper are available online. To view these files, please visit the journal online (http://dx.doi. org/10.1136/bmjopen-2018028302).

Received 6 December 2018 Revised 19 June 2019 Accepted 20 June 2019

Check for updates

(C) Author(s) (or their employer(s)) 2019. Re-use permitted under CC BY-NC. No commercial re-use. See rights and permissions. Published by BMJ.

For numbered affiliations see end of article.

Correspondence to Dr Camille Schwab; camille.schwab@aphp.fr

\section{ABSTRACT}

Objectives The aim of this systematic review was to describe and analyse the performance statistics of validated risk scores identifying elderly inpatients at risk of early unplanned readmission.

Data sources We identified potentially eligible studies by searching MEDLINE, EMBASE, COCHRANE and Web of Science. Our search was restricted to original studies, between 1966 and 2018.

Eligibility criteria Original studies, which internally or externally validated the clinical scores of hospital readmissions in elderly inpatients.

Data extraction and synthesis A data extraction grid based on Strengthening the Reporting of Observational Studies in Epidemiology and Transparent reporting of a multivariable prediction model for individual prognosis or diagnosis statements was developed and completed by two reviewers to collect general data. The same process was used to extract metrological data of the selected scores.

Quality assessment of the included

studies Assessment of the quality and risk of bias in individual studies was performed by two reviewers, using the validated Effective Public Health Practice Project quality assessment tool.

Participants Elderly inpatients discharged to home from hospital or returning home after an accident and emergency department visit.

Results A total of 12 studies and five different scores were included in the review. The five scores present area under the receiving operating characteristic curve between 0.445 and 0.69 . Identification of Senior At Risk (ISAR) and Triage Risk Screening Tool (TRST) scores were the more frequently validated scores with ISAR being more sensitive and TRST more specific.

Conclusions The TRST and ISAR scores have been extensively studied and validated. The choice of the most suitable score relies on available patient data, patient characteristics and the foreseen clinical care intervention. In order to pair the intervention with the appropriate clinical score, further studies of external validation of clinical scores, identifying elderly patients at risk of early unplanned readmission, are needed.

PROSPERO registration number CRD42017054516.
Strengths and limitations of this study

- Our systematic review includes clinical risk scores predicting elderly early unplanned readmission.

- Clinical risk scores give a numerical result and a cut-off, which might be more convenient for the current use.

- We identified potentially eligible studies by searching MEDLINE, EMBASE, COCHRANE and Web of Science.

- The data extraction grid is based on the Strengthening the Reporting of Observational Studies in Epidemiology and the Transparent reporting of a multivariable prediction model for individual prognosis or diagnosis statements.

\section{INTRODUCTION}

Hospital readmissions in the elderly are particularly deleterious, as they increase their risk of dependence and decompensation of comorbidities. ${ }^{1}$ Moreover, these readmissions are a burden for the health system organisations. Therefore, it has become a priority to decrease hospital readmission rates and for this purpose, unplanned hospital readmission, within 30 days after discharge, is now recognised as a valid quality indicator for hospital performance as these readmissions are likely to be connected to the index admission. ${ }^{2}$ Among all inpatients, the elderly are reportedly at higher risk of unplanned readmission: $17.3 \%$ of readmission for patients aged 85 years and older, versus $16.6 \%$ for patients under 64 years old. ${ }^{3}$ Thus, it is important to implement evidence-based clinical care interventions focused on reducing unplanned readmission of elderly patients. ${ }^{4}$ A time scale needs to be defined in order to measure potential benefits of such clinical care interventions. Moreover, this time 
limit has been introduced in guidelines or programmes dealing with elderly hospital readmissions in the USA and France. ${ }^{15}$

In order to better rationalise resources, healthcare authorities have stated that it is neither 'necessary nor efficient to intervene for every patient'. Moreover, as mono-faceted interventions only have a limited effect, ${ }^{6}$ care bundle interventions should be implemented. However, these interventions are costly in terms of time, money and caregiving and there is a need to determine who will benefit from those interventions in priority. ${ }^{1}$

In this vein, clinical risk scores, which are prognosis tools estimating the probability or risk of future conditions, ${ }^{7}$ can help physicians and pharmacists to identify elderly patients at high risk of unplanned readmission within 30 days after discharge. Building a clinical risk score requires three successive steps $^{8}:$ (1) the development; corresponding to the construction of the score on a derivation cohort, (2) an internal validation, that is, testing the score on the same population (validation cohort) and (3) an external validation, that is, adjusting or updating the score in other patients. Internal and external validation studies evaluate the discrimination of the scores, using the c-statistic, ${ }^{9}$ and several metrological characteristics such as specificity or sensitivity. In our study, the c-statistic of risk scores that identify elderly inpatients at risk of 30-day unplanned readmission, will give the probability a randomly selected readmitted patient had a higher score than a non-readmitted patient. The specificity will give the ability to reject patients at low to moderate risk and sensitivity the ability to correctly include patients at high risk of readmission. In 2011, Kansagara et $a l^{10}$ conducted a systematic review to identify risk prediction models for hospital readmission. This systematic review included thirty studies. Among the thirty studies, fourteen chose unplanned readmission between 31 days and 4years after discharge as outcome, six studies focused on specific conditions and seven focused on adult inpatients. We chose to exclude this kind of studies and to focus on elderly patients and all-cause unplanned readmissions within thirty days after discharge. The three remaining studies presented prediction models with no calculation of discrimination. We chose to include only studies for which the expression of discrimination was made by calculating the c-statistic or the area under the ROC curves. In 2016, while updating the review from Kansagara, Zhou et $\mathrm{ll}^{11}$ included a total of 60 studies in their systematic review. Among the 60 included studies, only four focused on elderly patients and all-cause unplanned readmissions within thirty days after discharge, two were prediction models and one was a clinical risk score without calculation of the discrimination ability.

Therefore, these studies have not enabled us to select a clinical score suitable for elderly inpatients at risk of unplanned readmission within 30 days after discharge.

The aim of this systematic review was to describe and analyse the statistical performances of validated risk scores identifying elderly inpatients at risk of 30 day unplanned readmission.

\section{METHODS}

This systematic review was registered in the PROSPERO database and is presented according to the Preferred Reporting Items for Systematic Reviews and Meta-Analyses guidelines (online supplementary file 1). ${ }^{12}$

\section{Eligibility criteria}

We have systematically identified peer-reviewed original studies, with internally or externally validated clinical scores of unplanned hospital readmissions in elderly inpatients. We excluded reviews and studies identifying readmission risk factors, developing a statistical prediction model for readmission and clinical score not having been, previously, internally validated. Indeed, statistical prediction models give a probability of a patient being readmitted, whereas clinical risk scores give a number which can be compared with a threshold from which the patient is at high risk of readmission. Clinical scores are, thus, easier to use routinely.

We included studies:

In which participants were elderly inpatients (65 years old and over) discharged to home after a hospitalisation or returning home after an accident and emergency department (AED) visit.

In which the main outcome measured was unplanned readmission or return to the AED within a month (28, 30 or 31 days) after discharge. We excluded studies that focused on specific conditions.

Where the score discrimination was evaluated using the c-statistic. The graphic representation of the c-statistic is the receiving operating characteristic (ROC) curve. We excluded studies which had used neither the c-statistic, nor the area under the curve (AUC) of the ROC curves for the validation.

\section{Information sources and search strategy}

We identified potentially eligible studies by searching in four databases MEDLINE EMBASE, COCHRANE and Web of Science. Our search was restricted to original human studies, published in French or English, between 1966 and 2017. The search strategies of the four databases were approved by a university librarian (online supplementary file 2). The electronic search was conducted on 13 January 2017 and updated on 19 January 2018. In addition to searching databases, we checked reference lists of selected studies and researched also the studies citing the selected studies, on Web of Science.

\section{Study selection}

After identification, studies were screened independently by two reviewers (CS and VK-S) on the basis of title and abstract. Then, the full text was read for potential inclusion in the systematic review and agreement between the two reviewing authors (CS and VK-S) was assessed using Cohen's $\kappa$ statistic. ${ }^{13} \mathrm{~A}$ Cohen's $\kappa$ coefficient comprised between 0.60 and 0.74 was considered as good, and very good if superior to 0.75 . In the case of disagreement, a third reviewer $(\mathrm{PH})$ was a priori designed to be consulted. 


\section{Data collection process}

We developed a data extraction grid based on The Strengthening the Reporting of Observational Studies in Epidemiology statement: guidelines for reporting observational studies ${ }^{14}$ and the Transparent reporting of a multivariable prediction model for individual prognosis or diagnosis (TRIPOD): the TRIPOD statement. ${ }^{15}$ General data extracted from full-text included: authors, year of publication, design, setting, participants, data sources, study size, quantitative variables and statistical analysis methods. For each selected study, CS extracted the general data and completed the grid. Independently, VKS checked all the data collected in the grid. Disagreements were resolved by discussion and consensus.

Using the same process, we extracted metrological data (AUC of the ROC curves, sensitivity, specificity, positive predictive value, negative predictive value, negative and positive likelihood ratio and accuracy) of the selected scores. Discrimination was considered fair for a c-statistic comprised between 0.5 and 0.69 , and good when the c-statistic was superior to 0.7 .

\section{Quality assessment}

Assessment of quality in individual studies was performed independently by two reviewers (CS and VK-S), using the validated Effective Public Health Practice Project (EPHPP) quality assessment tool. ${ }^{16}$ This instrument, usable for any quantitative study design, was adapted to our study design in order to evaluate the quality through five sections: selection bias, study design, data collection method, statistical analysis and withdrawals and dropouts. The quality was individually rated for each component (strong, moderate or weak) and added to assign a global rating to each study. The global rating followed the EPHPP guidelines: a strong global rating means there is no weak rating, a moderate global rating means there is one weak rating and a weak global rating means there are two or more weak ratings.

The quality across studies was assessed using a graphic representation of the percentage of studies with a strong, moderate or weak rating for each sections considered in the evaluation of the quality in individual studies.

\section{Patient and public involvement statement}

No patient was involved in this systematic review.

\section{RESULTS}

\section{Result of search strategy}

From the four databases, we extracted 2484 references (online supplementary file 3). After removal of 320 duplicates, we screened 2164 studies on the basis of titles and abstracts leading to the exclusion of 2075 irrelevant publications. A total of 93 publications were reviewed for eligibility. Nine studies finally met the inclusion criteria. We found three additional records by manual search (by checking the reference lists). Thus, a total of 12 studies $^{17-28}$ and five different scores were included in the present analysis. The degree of agreement between the two reviewers was good, with a $\kappa$ coefficient of 0.72 (95\% CI 0.50 to 0.93$)$.

\section{Study characteristics}

Study characteristics are presented in table 1 . The 12 included studies were published between 2000 and 2017. Six were conducted in Europe, ${ }^{17-2026} 27$ four in North America (the USA and Canada), ${ }^{21-2328}$ one in Asia (Singapore $)^{24}$ and one in Australia. ${ }^{25}$ Design, population, interventions and outcome will be detailed below.

\section{Study design}

All the studies were prospective observational cohort studies, except one which was a retrospective observational cohort study ${ }^{24}$ and one in which the design was not specified. ${ }^{27}$ Eight studies were monocentric ${ }^{17-2024-27}$ and four were multicentric. ${ }^{21-23} 28$

\section{Study population}

Participants were inpatients, 65 years old and over in 10 studies ${ }^{17-25} 28$ or 75 years old and over in one study. ${ }^{26}$ For one study, the age for inclusion was not mentioned, but patients were admitted to a geriatric ward. ${ }^{27}$ The mean age varies from 74 to 84.8 years old. Participants were discharged to home after either an AED visit, ${ }^{17}$ 19-23 252628 a hospitalisation ${ }^{24}$ or after an AED visit followed by a hospital admission. ${ }^{18}$ The readmission rate varies from $12.1 \%$ to $28.4 \%$

\section{Intervention}

Among the 12 studies, three internally validated a clinical score predicting hospital readmissions, ${ }^{22} 2325$ meaning that included patients constituted validation cohorts. The other nine studies consisted of an external score validation, meaning that the scores were tested in a new cohort, different from the one used for validation. The validation cohort studies of these scores included adult inpatients and thus, they were not included in the present review.

\section{Outcomes}

The definition of readmission differs between studies: it was either an AED visit, ${ }^{17} 192225$ an unplanned readmission $^{182427}$ or both (composite outcome) ${ }^{2021232628}$ within 1 month (from 28 to 30 days). For the Identification of Senior At Risk (ISAR) and Triage Risk Screening Tool (TRST) scores, the outcomes were an AED visit or a composite outcome, except for the Braes et alstudy ${ }^{18}$ where the outcome was an unplanned readmission. In this study, the c-statistic was lower than in the other studies $(0.445$ for the ISAR score and 0.478 for the TRST). Regarding the lengths of stay, admission, Charlson, emergency (LACE) index, the outcome was only an unplanned readmission. ${ }^{24} 27$

Because of the large heterogeneity of the studies (data sources and definition of readmission, table 1), a meta-analysis was not possible. 


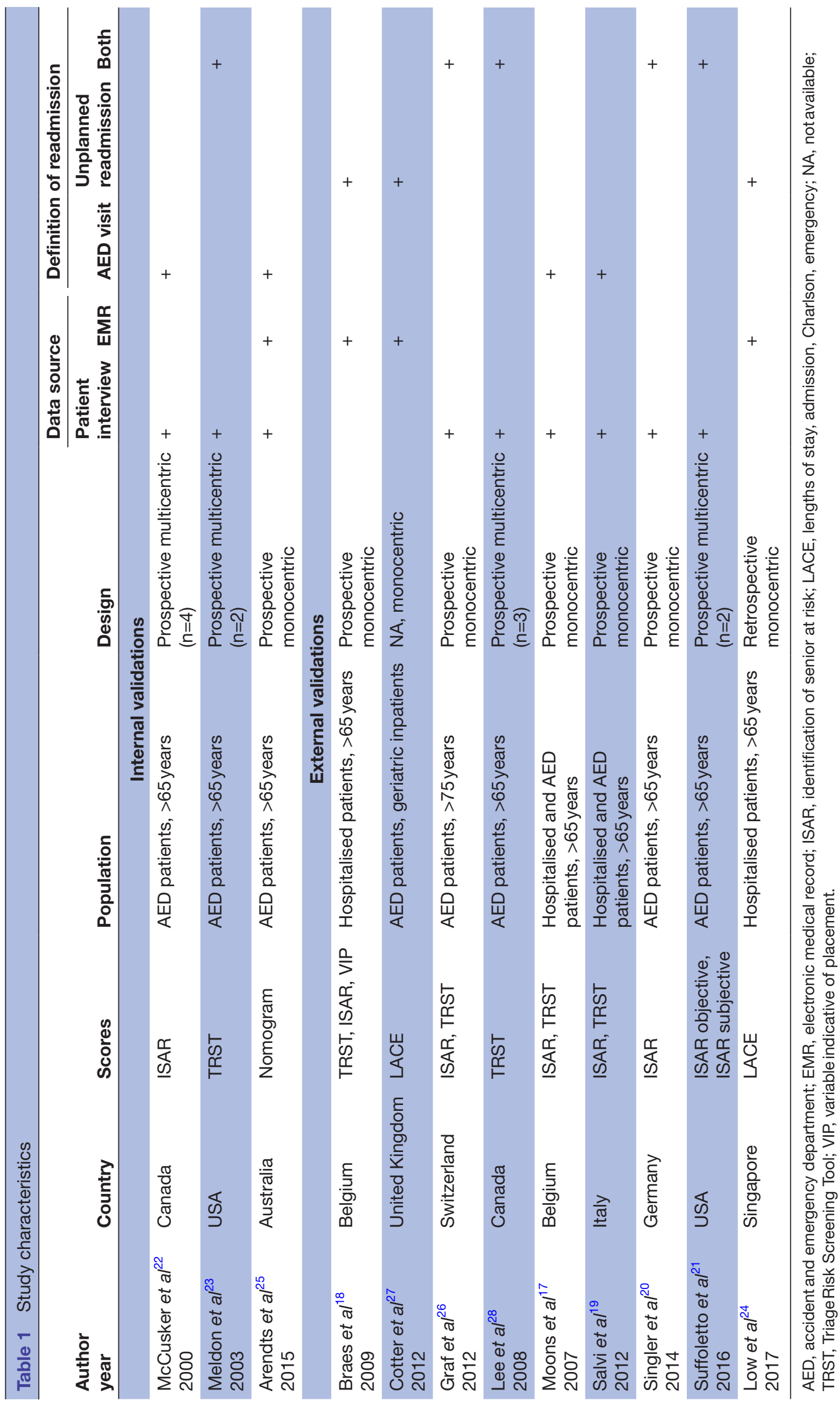




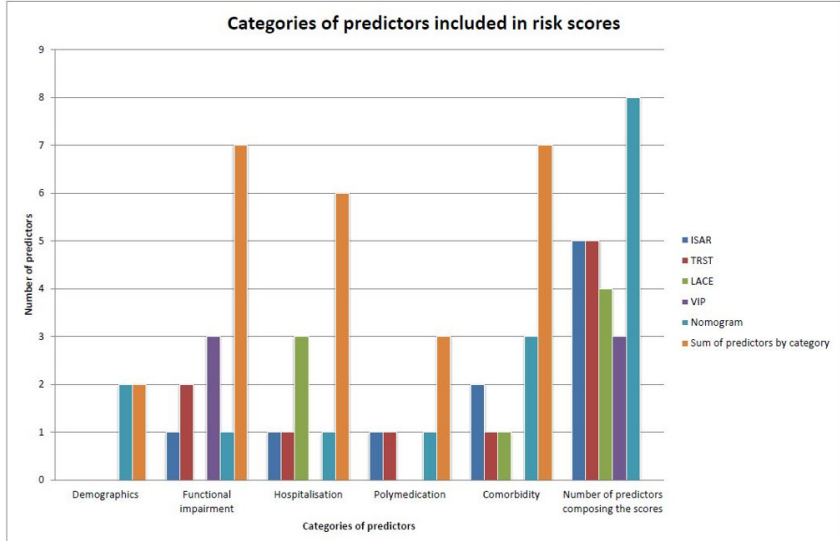

Figure 1 Categories of predictors included in risk scores.

\section{Scores characteristics}

The scores characteristics will be detailed bellow, by presenting predictors and measure of predictive performance (online supplementary files 4,5 and 6).

\section{Predictors}

The median number of items included in the scores was 5 (range 3-8). As depicted in figure 1, the predictors composing the five scores can be divided into five categories: demographics, functional impairment, hospitalisation, polymedication and comorbidity. Functional impairment and comorbidity are the most used predictors, whereas demographics are used in one score (nomogram). The assessment of each category differs between scores, eg, the functional impairment can be measured with the capacity to walk (TRST), the ability to use a phone (variable indicative of placement (VIP)) or the presence of a home carer (nomogram). Likewise, polymedication varies from more than three drugs to more than 6. All the categories are not use in every score, eg, the LACE index assesses readmission risk with hospitalisation characteristics (length of stay, admission type and history of AED visit) and comorbidity.

Each predictor can be obtained at admission, except for the length of stay (LACE index) which will be obtained at discharge.

Measure of predictive performance: As depicted in figure 2, the c-statistics for the ISAR score were close and comprised between 0.608 and 0.690, except for Braes $e t$ $a l$ study, ${ }^{18}$ whereas the c-statistics of the TRST score were scattered and comprised between 0.48 and 0.65 . The TRST score had a better discrimination (AUC of the ROC curve of 0.647 ) in the internal validation study than in the external validation studies. The three other scores (VIP, nomogram and LACE) have fair discriminations: the c-statistics were comprised between 0.5 and 0.65 , the nomogram having the best c-statistics (0.65). Moreover, the AUC of the ROC curves of the ISAR score were quite similar: 0.55 (95\% CI 0.49 to 0.61 ) and 0.595 (95\% CI 0.581 to 0.608$)$, respectively.

As depicted in figure 3, TRST had a higher specificity and a lower sensitivity - the sensitivities varied from 0.62 to 0.871 and the specificities from 0.215 to 0.63 -whereas

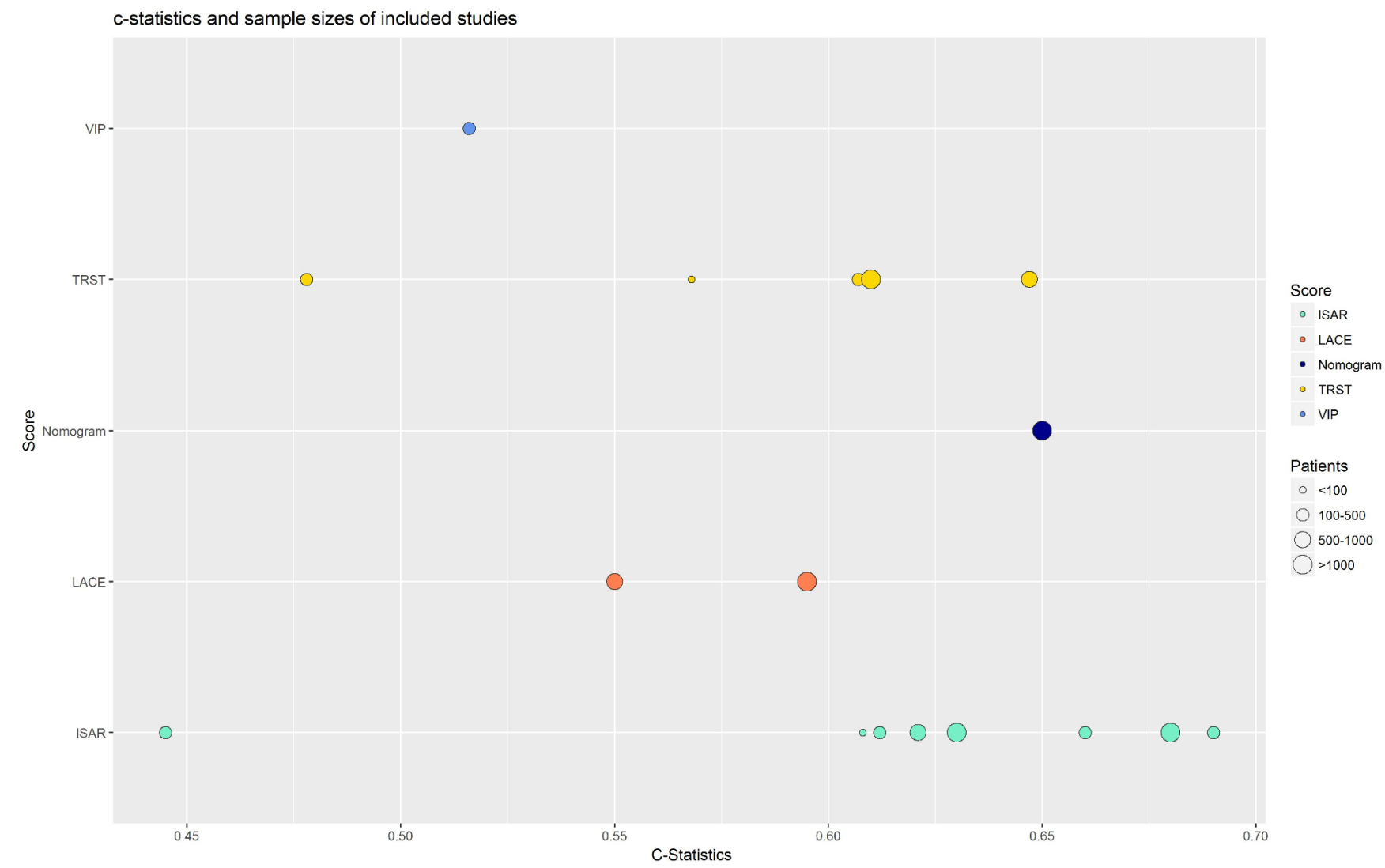

Figure 2 c-Statistics and sample sizes of included studies. 


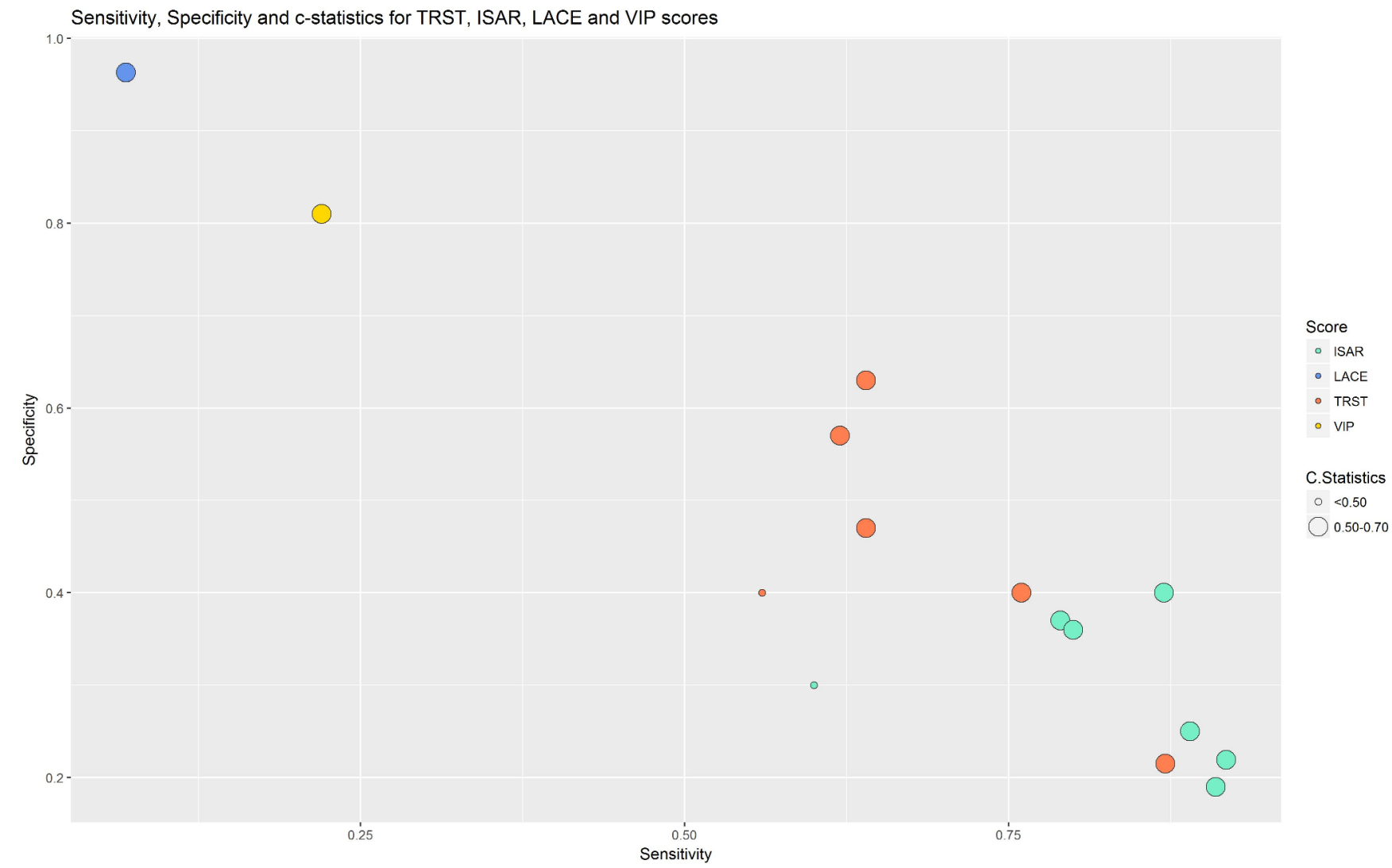

Figure 3 Sensitivity, specificity and c-statistics of the scores Identification of Senior At Risk (ISAR), lengths of stay, admission, Charlson, emergency (LACE), Triage Risk Screening Tool (TRST) and variable indicative of placement (VIP).

ISAR has a higher sensitivity and a lower specificity-the sensitivities varied from 0.79 to 0.918 and the specificities from 0.19 to 0.40 - and they showed good reproducibility. The two other scores, LACE and VIP, with only one study for each, showed better sensitivity and specificity than ISAR and TRST but had been externally validated only once. Finally, the specificity and sensitivity of the nomogram were not available.

In the external validation study by Braes et al, ${ }^{18}$ the VIP score showed an AUC of the ROC curve of 0.516, a sensitivity of 0.22 and a specificity of 0.81 (online supplementary file 6 ). The others metrological qualities are presented in the supplementary files, but they were not calculated for each score (online supplementary files 4-6).

The sensitivities and specificities were evaluated based on thresholds defined in the studies. Different thresholds could be defined to achieve different results for these two statistics.

\section{Quality assessment within studies}

All included studies were observational studies. Thus, we evaluated whether the study was prospective (low risk of bias) or retrospective (moderate risk of bias).

All the studies were at low risk of bias (prospective studies), except Low $e t a l^{24}$ which was a retrospective cohort study, and Cotter $e t a l,{ }^{27}$ where the design was not specified.
As we assume that multicentric studies have a more representative population than monocentric studies, we identified four studies with a low risk of bias (multicentric studies) $)^{21-2328}$ and eight studies with a moderate risk of bias (monocentric studies). ${ }^{17-20} 24-27$

To determine if patients had an unplanned readmission, the data collection for these observational studies was made from the medical records (high risk of bias), with the patient (moderate risk of bias) or both or with a national clinical data base (low risk of bias). The medical records rarely indicated if an unplanned readmission occurred in a different hospital, because about $20 \%$ of readmissions can be to a different hospital to the index admission. ${ }^{29}$ But the telephone interview and the national clinical data bases, they make it possible to overcome this classification bias.

We then evaluated whether the withdrawals and dropouts were reported in terms of number and/or reasons per group (low risk of bias) or not (high risk of bias). Four studies had not reported the withdrawals and dropouts. $^{20242528}$

We evaluated the quality of the statistical analysis as to whether the discrimination and the metrological data (low risk of bias), or only the discrimination (moderate risk of bias) were calculated.

Finally, we rated the global quality: three studies ${ }^{17} 1823$ had a global low risk of bias, seven ${ }^{19-22242627}$ had a moderate 

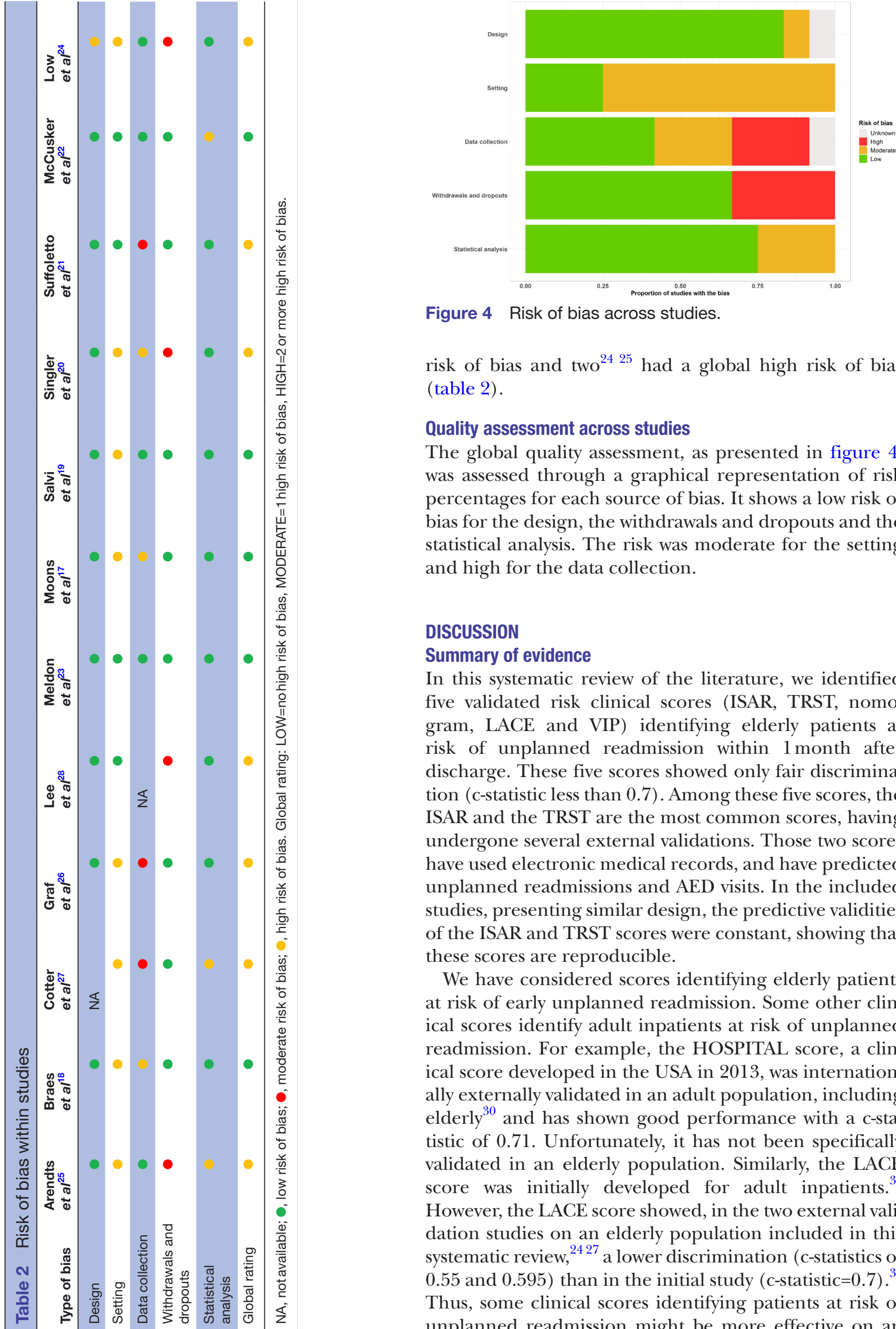

Figure 4 Risk of bias across studies.

risk of bias and two ${ }^{24} 25$ had a global high risk of bias (table 2).

\section{Quality assessment across studies}

The global quality assessment, as presented in figure 4, was assessed through a graphical representation of risk percentages for each source of bias. It shows a low risk of bias for the design, the withdrawals and dropouts and the statistical analysis. The risk was moderate for the setting and high for the data collection.

\section{DISCUSSION}

\section{Summary of evidence}

In this systematic review of the literature, we identified five validated risk clinical scores (ISAR, TRST, nomogram, LACE and VIP) identifying elderly patients at risk of unplanned readmission within 1 month after discharge. These five scores showed only fair discrimination (c-statistic less than 0.7). Among these five scores, the ISAR and the TRST are the most common scores, having undergone several external validations. Those two scores have used electronic medical records, and have predicted unplanned readmissions and AED visits. In the included studies, presenting similar design, the predictive validities of the ISAR and TRST scores were constant, showing that these scores are reproducible.

We have considered scores identifying elderly patients at risk of early unplanned readmission. Some other clinical scores identify adult inpatients at risk of unplanned readmission. For example, the HOSPITAL score, a clinical score developed in the USA in 2013, was internationally externally validated in an adult population, including elderly ${ }^{30}$ and has shown good performance with a c-statistic of 0.71 . Unfortunately, it has not been specifically validated in an elderly population. Similarly, the LACE score was initially developed for adult inpatients. ${ }^{31}$ However, the LACE score showed, in the two external validation studies on an elderly population included in this systematic review, ${ }^{24} 27$ a lower discrimination (c-statistics of 0.55 and 0.595$)$ than in the initial study $(c-s t a t i s t i c=0.7){ }^{31}$ Thus, some clinical scores identifying patients at risk of unplanned readmission might be more effective on an 
adult population, rather than on an elderly population. In a recent study, Cooksley $e t a l^{2}$ externally validated the LACE score in an adult inpatient population from two hospitals in Denmark. In this study, the authors evaluated the modification in discrimination of LACE score by age group and found that the discriminatory power of the LACE score decreased with increasing age. The authors also evaluated the change in discrimination of the HOSPITAL score, and came to the same conclusion: the discriminatory power of the HOSPITAL score decreases with increasing age.

We only included studies dealing with general elderly population. On the contrary, some clinical risk scores focus on specific condition. For example, Pack et $a l^{33}$ developed and validated a score predicting readmission after heart valve surgery and Echevarria $e t a l^{34}$ described a score predicting readmission after hospitalisation for acute exacerbation of chronic-obstructive pulmonary disease. These two scores predicted readmission with fair discrimination: c-statistic $=0.67$ and 0.70 , respectively. Echevarria $e t a l^{34}$ also externally validated the LACE score for the prediction of readmission after hospitalisation for acute exacerbation of chronic-obstructive pulmonary disease and found a c-statistic of 0.65. The LACE score was also externally validated to predict readmission in patients hospitalised with heart failure, and showed a fair discrimination, with a c-statistic of $0.59 .{ }^{35}$ Hence, clinical scores focusing on specific patient populations or general risk scores used on specific patient population have not shown a better discrimination than general scores used on general populations.

For the ISAR and TRST scores, the c-statistics seem to be related to the outcome: the c-statistics appeared, indeed, lower for a prediction of an unplanned readmission, compared with an AED visit or a composite outcome.

\section{Limitations}

Our systematic review has some limitations. Unlike the review by Kansagara et $a l,{ }^{10}$ we excluded risk prediction models, in order to include only clinical risk scores which might be more convenient for the current use. Clinical risk scores indeed give a numerical result and a cut-off, which could be automatically calculated by the medical software.

Moreover, we excluded validation studies on adult inpatients, which implies that studies with subanalyses that examine a score among the elderly population while the primary analyses were performed for a broad population were excluded. This is the case, for example, of the study of Cooksley et $a l^{32}$ which have shown that the discriminatory power of the LACE index and the HOSPITAL score ${ }^{30}$ decreased with increasing age.

Similarly, we excluded validation studies that focus on specific conditions or where the outcome was readmission within more than 1 month. These exclusion criteria have limited the number of scores presented in this review, whereas some scores have shown good discrimination ability, and thus might be interesting to externally validate in other conditions. For example, the $80+$ score, ${ }^{36}$ an internally validated score on an elderly population which focuses on the patients "prescription, predicts the readmission within 1 year, with a good discrimination (c-statistic $=0.72(95 \%$ CI 0.66 to 0.77$)$ ).

\section{CONCLUSIONS}

This systematic review showed that several validated scores identifying elderly patients at risk of early readmission are available. Among all scores, ISAR and TRST scores have been extensively studied and validated and three promising scores (VIP, nomogram and LACE) need further examination, even if the choice of the best fitting score to our patients depends on the available data, the patient and intervention characteristics. Their use can facilitate the implementation of bundle interventions on high risk patients, given the limited resources available.

Funding: This research received no specific grant from any funding agency in the public, commercial or not-forprofit sectors

Acknowledgements: The authors would like to acknowledge Drs Agnes deChartres and Germain Perrin for their informed advice for the writing of this systematic review, Sophie Guiquerro, university librarian of the University of Paris Descartes for her support developing the literature search strategy and Stella Ghouti for the correction of the manuscript.

Author Contributions: Concept, design: C. Schwab, V. Savoldelli and P. Hindlet. Data Collection: C. Schwab and V. Savoldelli. Interpretation of results: C. Schwab, V. Savoldelli, P. Hindlet and C. Fernandez. Drafting of manuscript: C. Schwab. Critical revision of manuscript for important intellectual content: B. Sabatier, C. Schwab, V. Savoldelli, P. Hindlet and C. Fernandez.

\section{Author affiliations}

1UMR-S 1136- Institut Pierre Louis D’Epidémiologie et de Santé Publique, Sorbonne Université, UPMC Univ Paris 06, Paris, France

${ }^{2}$ Service Pharmacie, Hôpital Européen Georges Pompidou, Assistance Publique Hôpitaux de Paris, Paris, France

${ }^{3}$ Service Pharmacie, Hôpital Saint-Antoine, Assistance Publique - Hôpitaux de Paris, Paris, France

${ }^{4}$ Faculté de Pharmacie, Université Paris-Sud, Paris, France

${ }^{5}$ INSERM UMR 1138 Team 22: Information Sciences to Support, Personalized Medicine, Cordelier Research Center, Paris, France

Correction notice This article has been corrected since it was published. The affiliations and acknowledgement section have been updated.

Acknowledgements The authors would like to acknowledge Dr Agnes Dechartres and Dr Germain Perrin for their informed advice for the writing of this systematic review, Sophie Guiquerro, university librarian of the University of Paris Descartes for her support in developing the literature search strategy and Stella Ghouti for the correction of the manuscript.

Contributors Concept, design: CS, VK-S and PH. Data collection: CS and VK-S. Interpretation of results: CS, VK-S, PH and CF. Drafting of manuscript: CS. Critical revision of manuscript for important intellectual content: BS, CS, VK-S, PH and CF.

Funding The authors have not declared a specific grant for this research from any funding agency in the public, commercial or not-for-profit sectors.

Competing interests None declared.

Patient consent for publication Not required. 
Provenance and peer review Not commissioned; externally peer reviewed.

Data sharing statement It is a systematic review in which all the available data are presented in the manuscript or in the supplementary files.

Open access This is an open access article distributed in accordance with the Creative Commons Attribution Non Commercial (CC BY-NC 4.0) license, which permits others to distribute, remix, adapt, build upon this work non-commercially, and license their derivative works on different terms, provided the original work is properly cited, appropriate credit is given, any changes made indicated, and the use is non-commercial. See: http://creativecommons.org/licenses/by-nc/4.0/.

\section{REFERENCES}

1. Haute Autorité de Santé. Comment réduire le risque de réhospitalisations évitables des personnes âgées ? 2013.

2. Fischer C, Lingsma HF, Marang-van de Mheen PJ, et al. Is the Readmission Rate a Valid Quality Indicator? A Review of the Evidence. PLoS ONE [Internet]. 2014;9 https://www.ncbi.nlm.nih. gov/pmc/articles/PMC4224424/.

3. Barnett ML, Hsu J, McWilliams JM. Patient Characteristics and Differences in Hospital Readmission Rates. JAMA Intern Med 2015;175:1803-12.

4. Legrain S, Tubach F, Bonnet-Zamponi D, et al. A new multimodal geriatric discharge-planning intervention to prevent emergency visits and rehospitalizations of older adults: the optimization of medication in AGEd multicenter randomized controlled trial. J Am Geriatr Soc 2011;59:2017-28.

5. Mcllvennan CK, Eapen ZJ, Allen LA. Hospital readmissions reduction program. Circulation 2015;131:1796-803.

6. Hansen LO, Young RS, Hinami K, et al. Interventions to reduce 30-day rehospitalization: a systematic review. Ann Intern Med 2011;155:520-8.

7. Moons KG, Royston P, Vergouwe $\mathrm{Y}$, et al. Prognosis and prognostic research: what, why, and how? BMJ 2009;338:b375.

8. Moons KG, Kengne AP, Grobbee DE, et al. Risk prediction models: II. External validation, model updating, and impact assessment. Heart 2012;98:691-8.

9. Han K, Song K, Choi BW. How to Develop, Validate, and Compare Clinical Prediction Models Involving Radiological Parameters: Study Design and Statistical Methods. Korean J Radiol 2016;17:339-50.

10. Kansagara D, Englander H, Salanitro A, et al. Risk prediction models for hospital readmission: a systematic review. JAMA 2011;306:1688-98.

11. Zhou H, Della PR, Roberts P, et al. Utility of models to predict 28-day or 30-day unplanned hospital readmissions: an updated systematic review. BMJ Open 2016;6:e011060.

12. Liberati A, Altman DG, Tetzlaff $\mathrm{J}$, et al. The PRISMA statement for reporting systematic reviews and meta-analyses of studies that evaluate health care interventions: explanation and elaboration. $J$ Clin Epidemiol 2009;62:e1-e34.

13. Cohen J. A coefficient of agreement for nominal scales. Educ Psychol Meas 1960;20:37-46.

14. von Elm E, Altman DG, Egger M, et al. The Strengthening the Reporting of Observational Studies in Epidemiology (STROBE) statement: guidelines for reporting observational studies. Ann Intern Med 2007;147:573.

15. Collins GS, Reitsma JB, Altman DG, et al. Transparent reporting of a multivariable prediction model for individual prognosis or diagnosis (TRIPOD): the TRIPOD statement. BMJ 2014;350:g7594.

16. Thomas BH, Ciliska D, Dobbins $\mathrm{M}$, et al. A process for systematically reviewing the literature: providing the research evidence for public health nursing interventions. Worldviews Evid Based Nurs 2004;1:176-84.

17. Moons P, De Ridder K, Geyskens K, et al. Screening for risk of readmission of patients aged 65 years and above after discharge from the emergency department: predictive value of four instruments. Eur J Emerg Med 2007;14:315-23.

18. Braes T, Moons P, Lipkens P, et al. Screening for risk of unplanned readmission in older patients admitted to hospital: predictive accuracy of three instruments. Aging Clin Exp Res 2010;22:345-51.

19. Salvi F, Morichi V, Lorenzetti B, et al. Risk stratification of older patients in the emergency department: comparison between the Identification of Seniors at Risk and Triage Risk Screening Tool. Rejuvenation Res 2012;15:288-94.

20. Singler K, Heppner HJ, Skutetzky A, et al. Predictive validity of the identification of seniors at risk screening tool in a German emergency department setting. Gerontology 2014;60:413-9.

21. Suffoletto B, Miller T, Shah R, et al. Predicting older adults who return to the hospital or die within 30 days of emergency department care using the ISAR tool: subjective versus objective risk factors. Emerg Med J 2016;33:4-9.

22. McCusker J, Cardin S, Bellavance F, et al. Return to the emergency department among elders: patterns and predictors. Acad Emerg Med 2000;7:249-59.

23. Meldon SW, Mion LC, Palmer RM, et al. A brief risk-stratification tool to predict repeat emergency department visits and hospitalizations in older patients discharged from the emergency department. Acad Emerg Med 2003;10:224-32.

24. Low LL, Liu N, Meh O, et al. Performance of the LACE index to identify elderly patients at high risk for hospital readmission in Singapore. Medicine (Baltimore) [Internet]. 2017;96 https://www.ncbi. nlm.nih.gov/pmc/articles/PMC5428584/.

25. Arendts G, Etherton-Beer $\mathrm{C}$, Jones $\mathrm{R}$, et al. Use of a risk nomogram to predict emergency department reattendance in older people after discharge: a validation study. Intern Emerg Med 2015;10:481-7.

26. Graf CE, Giannelli SV, Herrmann FR, et al. Identification of older patients at risk of unplanned readmission after discharge from the emergency department - comparison of two screening tools. Swiss Med Wkly 2012;141:w13327.

27. Cotter PE, Bhalla VK, Wallis SJ, et al. Predicting readmissions: poor performance of the LACE index in an older UK population. Age Ageing 2012;41:784-9.

28. Lee JS, Schwindt G, Langevin M, et al. Validation of the triage risk stratification tool to identify older persons at risk for hospital admission and returning to the emergency department. J Am Geriatr Soc 2008:56:2112-7.

29. Nasir K, Lin Z, Bueno $\mathrm{H}$, et al. Is same-hospital readmission rate a good surrogate for all-hospital readmission rate? Med Care 2010;48:477-81.

30. Donzé J, Aujesky D, Williams D, et al. Potentially avoidable 30-day hospital readmissions in medical patients: derivation and validation of a prediction model. JAMA Intern Med 2013;173:632-8.

31. van Walraven C, Dhalla IA, Bell C, et al. Derivation and validation of an index to predict early death or unplanned readmission after discharge from hospital to the community. Can Med Assoc $J$ 2010;182:551-7.

32. Cooksley T, Nanayakkara PW, Nickel $\mathrm{CH}$, et al. Readmissions of medical patients: an external validation of two existing prediction scores. QJM 2016;109:245-8.

33. Pack QR, Priya A, Lagu T, et al. Development and Validation of a Predictive Model for Short- and Medium-Term Hospital Readmission Following Heart Valve Surgery. J Am Heart Assoc 2016;5.

34. Echevarria C, Steer J, Heslop-Marshall K, et al. The PEARL score predicts 90-day readmission or death after hospitalisation for acute exacerbation of COPD. Thorax 2017;72:686-93.

35. Yazdan-Ashoori P, Lee SF, Ibrahim Q, et al. Utility of the LACE index at the bedside in predicting 30-day readmission or death in patients hospitalized with heart failure. Am Heart J 2016;179:51-8.

36. Alassaad A, Melhus $\mathrm{H}$, Hammarlund-Udenaes M, et al. A tool for prediction of risk of rehospitalisation and mortality in the hospitalised elderly: secondary analysis of clinical trial data. BMJ Open 2015;5:e007259. 